\title{
Achievement of Ultralow Emittance Beam in the Accelerator Test Facility Damping Ring
}

\author{
Y. Honda, ${ }^{1}$ K. Kubo, ${ }^{2}$ S. Anderson, ${ }^{3}$ S. Araki, ${ }^{2}$ K. Bane, ${ }^{3}$ A. Brachmann, ${ }^{3}$ J. Frisch, ${ }^{3}$ M. Fukuda, ${ }^{6}$ K. Hasegawa, ${ }^{14}$ \\ H. Hayano, ${ }^{2}$ L. Hendrickson, ${ }^{3}$ Y. Higashi, ${ }^{2}$ T. Higo, ${ }^{2}$ K. Hirano, ${ }^{13}$ T. Hirose, ${ }^{15}$ K. Iida, ${ }^{12}$ T. Imai, ${ }^{9}$ Y. Inoue, ${ }^{7}$ P. Karataev, ${ }^{6}$ \\ M. Kuriki, ${ }^{2}$ R. Kuroda, ${ }^{8}$ S. Kuroda, ${ }^{2}$ X. Luo, ${ }^{11}$ D. McCormick, ${ }^{3}$ M. Matsuda, ${ }^{10}$ T. Muto, ${ }^{2}$ K. Nakajima, ${ }^{2}$ Takashi Naito, ${ }^{2}$ \\ J. Nelson, ${ }^{3}$ M. Nomura, ${ }^{13}$ A. Ohashi, ${ }^{6}$ T. Omori, ${ }^{2}$ T. Okugi, ${ }^{2}$ M. Ross, ${ }^{3}$ H. Sakai, ${ }^{12}$ I. Sakai, ${ }^{13}$ N. Sasao, ${ }^{1}$ S. Smith, ${ }^{3}$ \\ Toshikazu Suzuki, ${ }^{2}$ M. Takano, ${ }^{13}$ T. Taniguchi, ${ }^{2}$ N. Terunuma, ${ }^{2}$ J. Turner, ${ }^{3}$ N. Toge, ${ }^{2}$ J. Urakawa, ${ }^{2}$ V. Vogel, ${ }^{2}$ \\ M. Woodley, ${ }^{3}$ A. Wolski, ${ }^{4}$ I. Yamazaki, ${ }^{8}$ Yoshio Yamazaki, ${ }^{2}$ G. Yocky, ${ }^{3}$ A. Young, ${ }^{3}$ and F. Zimmermann ${ }^{5}$ \\ ${ }^{1}$ Department of Physics, Kyoto University, Kyoto, Japan \\ ${ }^{2}$ KEK, High Energy Accelerator Research Organization, Tsukuba, Japan \\ ${ }^{3}$ Stanford Linear Accelerator Center (SLAC), Stanford, California, USA, 94024 \\ ${ }^{4}$ Lawrence Berkeley National Laboratory (LBNL), Berkeley, California, USA, 94720 \\ ${ }^{5}$ European Organization for Nuclear Research (CERN), Geneva, Switzerland \\ ${ }^{6}$ Department of Physics, Tokyo Metropolitan University, Hachioji, Japan \\ ${ }^{7}$ Department of Applied Physics, Tohoku-Gakuin University, Tagajo, Japan \\ ${ }^{8}$ Advanced Research Institute for Science and Engineering, Waseda University, Tokyo, Japan \\ ${ }^{9}$ Department of Physics, Faculty of Science and Technology, Science University of Tokyo, Chiba, Japan \\ ${ }^{10}$ Department of Physics, Faculty of Engineering, Yokohama National University, Yokohama, Japan \\ ${ }^{11}$ Institute of High Energy Physics (IHEP), Beijing, People's Republic of China \\ ${ }^{12}$ Institute for Solid State Physics, University of Tokyo, Chiba, Japan \\ ${ }^{13}$ National Institute of Radiological Sciences (NIRS), Chiba, Japan \\ ${ }^{14}$ The Graduate University for Advanced Studies, Tsukuba, Japan \\ ${ }^{15}$ Nagoya University, Nagoya, Japan \\ (Received 5 August 2003; published 6 February 2004)
}

\begin{abstract}
For high luminosity in electron-positron linear colliders, it is essential to generate low vertical emittance beams. We report on the smallest vertical emittance achieved in single-bunch-mode operation of the Accelerator Test Facility, which satisfies the requirement of the x-band linear collider. The emittances were measured with a laser-wire beam-profile monitor installed in the damping ring. The bunch length and the momentum spread of the beam were also recorded under the same conditions. The smallest vertical rms emittance measured at low intensity is $4 \mathrm{pm}$ at a beam energy of $1.3 \mathrm{GeV}$, which corresponds to the normalized emittance of $1.0 \times 1.0^{-8} \mathrm{~m}$. It increases by a factor of 1.5 for a bunch intensity of $10^{10}$ electrons. The measured data agreed to the calculation of intrabeam scattering within much better than a factor of 2 .
\end{abstract}

DOI: 10.1103/PhysRevLett.92.054802

For high luminosity in electron-positron linear colliders, it is essential to generate low vertical emittance beams. The damping ring of the Accelerator Test Facility (ATF) [1] at KEK has been designed to produce a beam with an extremely low emittance. The beam energy is $1.3 \mathrm{GeV}$. The natural, normalized horizontal rms emittance is $2.8 \times 10^{-6} \mathrm{~m}$, corresponding physical emittance is $1.1 \mathrm{~nm}$. For the $\mathrm{x}$-band linear collider, the normalized vertical emittance from the damping ring is required to be $2.0 \times 10^{-8} \mathrm{~m}$ with the bunch intensity of $7.5 \times 10^{9}$ electrons [2], which corresponds to the physical emittance of $8 \mathrm{pm}$ in the ATF ring.

Recently, several essential improvements were implemented which permitted us to further reduce the beam emittance: (1) The readout circuits for the beam position monitors (BPMs) in the damping ring were replaced by a new type [3]. This improved the position resolution to $2 \mu \mathrm{m}$. (2) The position offsets of the BPMs with respect to the field center of the nearest magnet were measured by a beam-based method [4]. (3) In January 2003, the mag-
PACS numbers: 29.27.Bd, 29.27.Fh, 41.75.Ht, 41.85.Ew

nets in the damping ring were realigned. (4) In parallel, the optics diagnostics and correction were further refined. They are based on measuring the beam-orbit response to corrector-magnet excitations.

For an accurate and reliable measurement of the beam emittances, the laser-wire beam-profile monitor was upgraded as well [5-7]. Two laser-wire systems are now available, which measure both the vertical and the horizontal beam size. The width of the horizontal wire was reduced, to be able to measure smaller electron beams. The effective laser power was increased by about 1 order of magnitude to shorten the data-acquisition time.

This Letter reports on the generation of an ultralow emittance electron beam [8], which satisfies the requirement of present design of a linear collider, in single-bunch operation mode at the ATF.

Low emittance tuning.-Our tuning method of the damping ring for low vertical emittance consists of a series of corrections [9-11]: COD (closed-orbit distortion) correction, followed, first, by a combined correction 
of vertical COD and dispersion and, finally, by a coupling correction. Because the vertical emittance in the damping ring is primarily determined by the vertical dispersion and by the horizontal-vertical coupling, it is essential to make the vertical dispersion and the coupling small. After the above corrections, the rms value of $\eta_{y \text {,meas }}$ is about $1.5 \mathrm{~mm}$ and the coupling, $\left[\Sigma_{\mathrm{BPM}}\left(\Delta y_{\text {steer }}\right)^{2} /\right.$ $\left.\Sigma_{\mathrm{BPM}}\left(\Delta x_{\text {steer }}\right)^{2}\right]$, about 0.004 , where $\Delta x_{\text {steer }}$ and $\Delta y_{\text {steer }}$ are the measured horizontal and vertical position responses to a set of horizontal steering magnets.

We observed that the residual COD, vertical dispersion, and $x-y$ coupling have been significantly reduced after the improvement of BPM readout circuits, by performing a beam-based offset correction of the vertical BPM readings, and with frequent beam-based updates of the optics model. For example, Fig. 1 shows the vertical dispersion measured on November 26, 2002, before the improvement, and that measured on May 20, 2003, after the improvement. Both measurements were performed after the combined COD and dispersion correction. The error bars were calculated from the fluctuations of the BPM readings. Evidently, both the residual vertical dispersion and the fluctuation became smaller.

While the actual ring tuning is carried out, the momentum spread of the beam is monitored. When the bunch volume is reduced, the momentum spread increased due to the intrabeam scattering. The dispersion correction and the coupling correction are iterated, until no further enlargement of the momentum spread is seen.

Beam diagnostics at the ATF.-Transverse emittances.-The transverse beam sizes in the damping ring were measured with a laser-wire monitor [7].
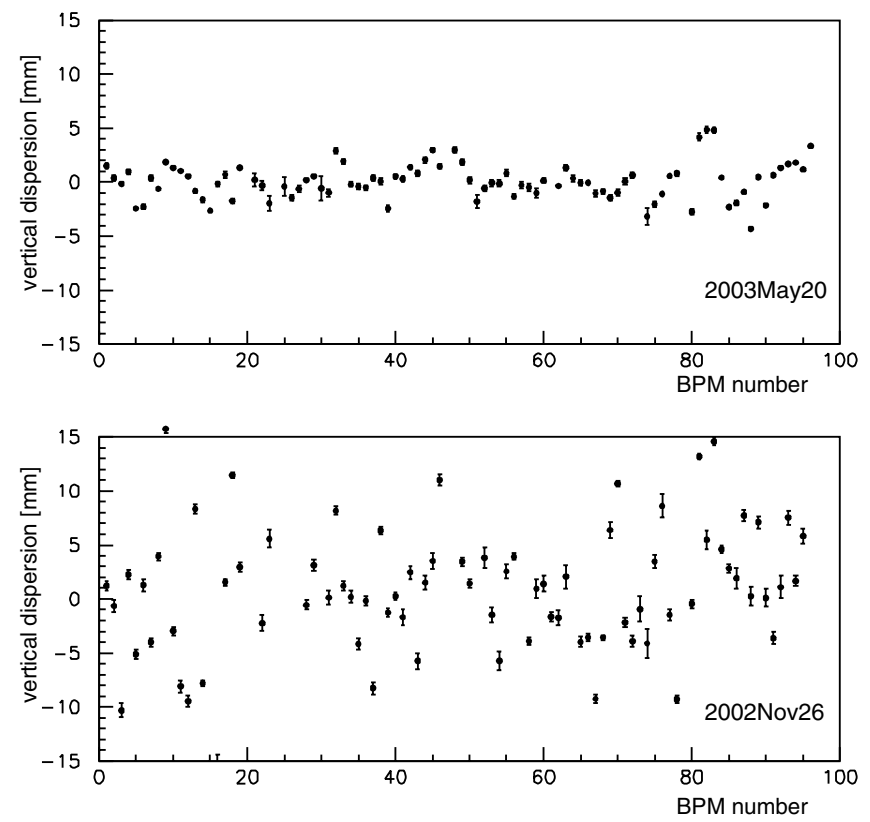

FIG. 1. Vertical dispersion measured by BPMs in the damping ring, after dispersion correction. Top: May 20, 2003; bottom: November 26, 2002.
Both electron beam and laser beam have a nearly Gaussian intensity distribution (with rms widths $\sigma_{e}$ and $\sigma_{l w}$, respectively ) in the transverse direction. The size measured $\left(\sigma_{\text {meas }}\right)$ is a convolution of the two. The real electron-beam size was extracted by unfolding the laser size as $\sigma_{e}=\sqrt{\sigma_{\text {meas }}^{2}-\sigma_{l w}^{2}}$.

There were three methods to estimate the width of laser wire (detail in [5,7]). The results were consistent with each other. The combined result for $\sigma_{l w}(h$ wire) was $5.65 \pm 0.08 \mu \mathrm{m}$ and $\sigma_{l w}(v$ wire $)$ was $14.7 \pm 0.2 \mu \mathrm{m}$.

The actual beam-size measurements were done as follows. An electron beam with a target intensity was stored in the damping ring. The position of the laser wire (the whole optical system on the table) was scanned in steps of $10 \mu \mathrm{m}$, and data were taken over $10 \mathrm{~s}$ per point for the vertical measurement, and every $50 \mu \mathrm{m}$ over $30 \mathrm{~s}$ for the horizontal.

One profile measurement corresponds to a complete scan back and forth across the beam (round-trip). Since the electron-beam current decreased during the measurement, the data taking was sometimes interrupted to reinject the electron beam, before resuming the scan. It took about 6 min to obtain one profile for the vertical beam-size measurement, and $15 \mathrm{~min}$ for the horizontal.

It should be noted that the obtained profile includes the amplitude of the beam-orbit jitter faster than $\sim 0.1 \mathrm{~Hz}$. The real size might be a little smaller especially in the vertical beam-size measurements for the small emittance cases.

The beam emittances were evaluated from $\epsilon \beta=\sigma_{e}^{2}-$ $[\eta(\Delta p / p)]^{2}$, where $\beta$ is the beta function, $\eta$ is the dispersion, and $\Delta p / p$ is the momentum spread of the beam. To estimate the beta function at the laser-wire positions, we measured the dependence of the betatron tunes on the strength of the quadrupoles in the neighborhood, from which we obtained the beta functions at the quadrupole position. The beta functions at the laser-wire position were inferred by interpolation. The dispersion function at the laser-wire position was estimated from the variation of the profile-center position induced by a slight change of the damping-ring rf frequency. It was found to be less than $2 \mathrm{~mm}(20 \mathrm{~mm})$ in the vertical (horizontal) plane. The dispersion term was found to be negligible and was ignored in the calculation.

Momentum spread.-The beam momentum spread was measured with the extracted beam. A screen monitor in a high dispersion region of the extraction line was used for this purpose. The horizontal beam size $\sigma_{x}$ at this screen was dominated by the dispersion term, so that $\Delta p / p=$ $\sigma_{x} / \eta_{x}$. The dispersion function at the screen monitor was measured to be $\eta_{x}=1.79 \mathrm{~m}$. The beam size on the screen $\left(\sigma_{x}\right)$ was estimated by fitting the profile image data with a Gaussian function.

Bunch length.-The bunch length $\left(\sigma_{z}\right)$ of the beam was obtained by using a streak camera to measure the time 
TABLE I. The ring conditions.

\begin{tabular}{ccc}
\hline \hline Run & Date & Status of coupling correctors \\
\hline A & $2 / 26$ & Normal $^{\text {a }}$ \\
B & $4 / 16$ & Normal $^{\text {a }}$ \\
C & $5 / 15$ & Half of them were turned off $^{\text {D }}$ \\
D & $5 / 20$ & Normal $^{\text {a }}$ \\
E & $5 / 23(1)$ & Half turned off, half reversed \\
F & $5 / 23(2)$ & All of them were turned off \\
\hline \hline
\end{tabular}

${ }^{a}$ Damping ring tuning was performed just before the measurement.

structure of the synchrotron radiation from one of the bending magnets in the arcs. The images obtained by the streak camera were fitted with a Gaussian function.

Results.-We measured the current dependence of the horizontal and vertical emittances, the beam momentum spread, and the bunch length under several conditions (or tuning levels) of the damping ring. The conditions of the ring in this series of experiments are listed in Table I. To change (enlarge) the vertical emittance, the coupling corrector trim coils in some sections of the ring were turned off or set to the reverse current, which enhances the horizontal-vertical coupling.

The beta functions at the laser-wire position were measured every day. Typically, $\beta_{y}$ at the horizontal laser wire was $4.51 \mathrm{~m}$, and $\beta_{x}$ at the vertical laser wire was $7.45 \mathrm{~m}$. These values were stable within $10 \%$ in this series of experiments.

At several different bunch populations up to about $1 \times$ $10^{10}$, the horizontal and the vertical emittances were measured by the laser-wire monitor. The emittance at bunch population $N$ is fitted by a simple empirical function which reproduces the result of the calculation by the SAD program [12], $\epsilon(N)=a \ln (b N+c)$, with three free parameters $(a, b$, and $c)$. From this fit result, we extracted two values, $\epsilon_{0}=a \ln (c)$ : emittance at zero intensity and $r_{10}=\ln \left(b 10^{10}+c\right) / \ln (c)$; emittance at $N=10^{10}$ inten- sity normalized by $\epsilon_{0}$ (strength of the current dependence). The results for each run are listed in Table II.

Data of runs $B$ and $D$ (normal condition, just after tuning) are shown in Figs. 2 and 3. The results of the momentum spread and the bunch length for some damping-ring conditions are shown in Figs. 4 and 5.

The source of the intensity dependence of the transverse emittance and the momentum spread is considered to be intrabeam scattering. The higher the particle density (smaller vertical emittance), the stronger is the effect of intrabeam scattering. This is clearly seen in the momentum-spread data though it is less evident in the transverse emittance. On the other hand, the intensity dependence of the bunch length is strong even when the vertical emittance is large. This last dependence is thus considered to be due to a combination of both intrabeam scattering and longitudinal wake fields.

The intensity dependence was compared with calculations using the SAD program. Since the impedance effects are not directly included in SAD, the inferred change of the bunch length due to the longitudinal impedance was simulated by changing the ring rf voltage ( $\mathrm{Vc})$, assuming that the impedance is purely inductive. To reproduce the measured bunch length data of runs $D, E$, and $F$, the impedance effect (reduction of $\mathrm{Vc}$ in the calculation) was fitted as a linear function of $N / \sigma_{z}^{3}$, where $\sigma_{z}$ is the rms bunch length. The results of these calculations are shown as lines in Figs. 2-5, where the vertical-horizontal emittance ratio was set, based on the measured data, as $0.4 \%$, $6 \%$, and $3 \%$ for the case of normal coupling correction (runs $B$ and $D$ ), for the case that half of the correctors were off and half reversed (run $E$ ) and for all off (run $F$ ), respectively. The intensity dependences of the emittance data for the other runs were also compared with calculations. The measured data agreed to the calculation of intensity dependences from intrabeam scattering within much better than a factor of 2 .

It should be noted that the results presented here differ from our old data reported in Ref. [9], where all the

TABLE II. Summary of the current dependence of the transverse emittances. Here, $\epsilon_{0}$ is the zero-current emittance, $r_{10}$ is the relative strength of the current dependence. Each value has two errors. The first error is from the data quality of the beam profile scan: the statistical error of the count rate of the gamma rays and the reproducibilities of the Gaussian beam profiles obtained in one round-trip. The second error is the systematic error from the uncertainty of the beta function and the width of the laser beam. This second error affects the conversion of $\sigma_{\text {meas }}$ into $\epsilon$ for all data of one day. The chi square per degree of freedom $\left(\chi^{2} / \mathrm{ndf}\right)$ of the fitting is also listed. We suspect the large $\chi^{2} /$ ndf variation comes from beam condition change during the measurement, such as a drift of the beam orbit, which is not included in the error estimation.

\begin{tabular}{|c|c|c|c|c|c|c|}
\hline Run & $\epsilon_{0 x}\left(10^{-9} \mathrm{~m} \mathrm{rad}\right)$ & $\begin{array}{l}\text { Horizontal } \\
r_{10 x}\left[=\epsilon\left(N=10^{10}\right) / \epsilon(N=0)\right]\end{array}$ & $\chi^{2} / \mathrm{ndf}$ & $\epsilon_{0 y}\left(10^{-12} \mathrm{~m} \mathrm{rad}\right)$ & $\begin{array}{l}\text { Vertical } \\
r_{10 y}\left[=\epsilon\left(N=10^{10}\right) / \epsilon(N=0)\right]\end{array}$ & $\chi^{2} / \mathrm{ndf}$ \\
\hline A & $1.08 \pm 0.09 \pm 0.03$ & $1.51 \pm 0.18 \pm 0.00$ & 1.26 & $6.65 \pm 0.63 \pm 0.35$ & $1.45 \pm 0.17 \pm 0.01$ & 0.195 \\
\hline B & $1.05 \pm 0.07 \pm 0.05$ & $1.46 \pm 0.15 \pm 0.00$ & 1.09 & $4.04 \pm 0.64 \pm 0.21$ & $1.53 \pm 0.34 \pm 0.03$ & 3.64 \\
\hline $\mathrm{C}$ & $1.01 \pm 0.11 \pm 0.12$ & $1.55 \pm 0.16 \pm 0.02$ & 0.215 & $16.39 \pm 1.35 \pm 0.69$ & $1.73 \pm 0.19 \pm 0.01$ & 1.44 \\
\hline $\mathrm{D}$ & $0.94 \pm 0.31 \pm 0.06$ & $1.88 \pm 0.64 \pm 0.01$ & / & $3.80 \pm 0.51 \pm 0.30$ & $1.23 \pm 0.26 \pm 0.02$ & 2.21 \\
\hline $\mathrm{E}$ & $1.12 \pm 0.14 \pm 0.02$ & $1.31 \pm 0.21 \pm 0.01$ & 1.49 & $68.74 \pm 6.78 \pm 2.29$ & $1.27 \pm 0.19 \pm 0.00$ & 0.721 \\
\hline $\mathrm{F}$ & $1.23 \pm 0.14 \pm 0.02$ & $1.31 \pm 0.19 \pm 0.00$ & 0.181 & $42.60 \pm 4.19 \pm 1.43$ & $1.13 \pm 0.15 \pm 0.00$ & 1.22 \\
\hline
\end{tabular}




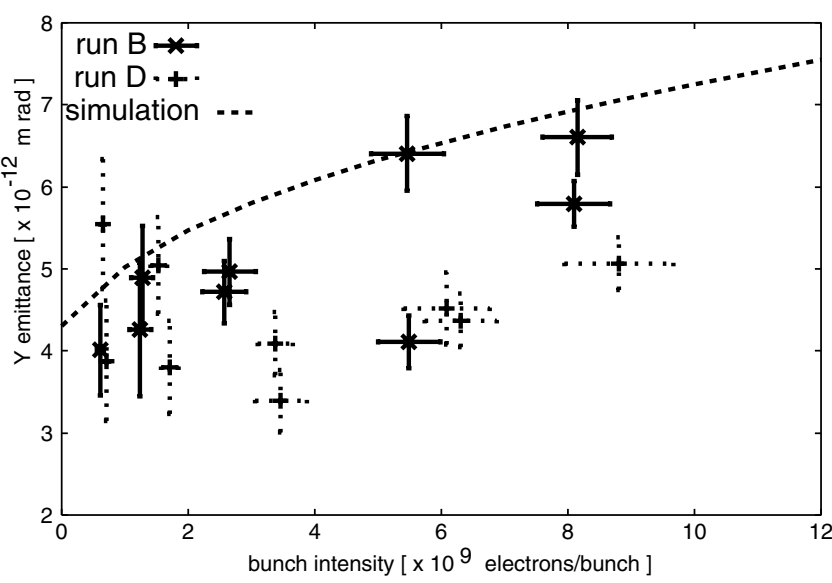

FIG. 2. Current dependence of the vertical emittance: Data for the smallest emittance cases (runs B and D) are shown. The result of a SAD simulation for $0.4 \%$ coupling is superimposed.

horizontal and most of the vertical emittance measurements had been performed using conventional wire scanners in the extraction line. Before, there was a much stronger emittance current dependence. The difference in behavior is not understood, though we suspect that unknown magnetic-field errors or/and beam losses at some components of the extraction line had affected these earlier measurements.

In this Letter, we have presented the achievement of the smallest vertical beam emittance in the damping ring of the ATF. The improvements on the readout circuit of the beam position monitors in the damping ring, the beambased correction of BPM offsets, and the optics diagnostics involving beam-based methods all proved essential for this ATF milestone. The transverse emittances were measured with an upgraded laser-wire monitor. The smallest vertical rms emittance measured at low intensity

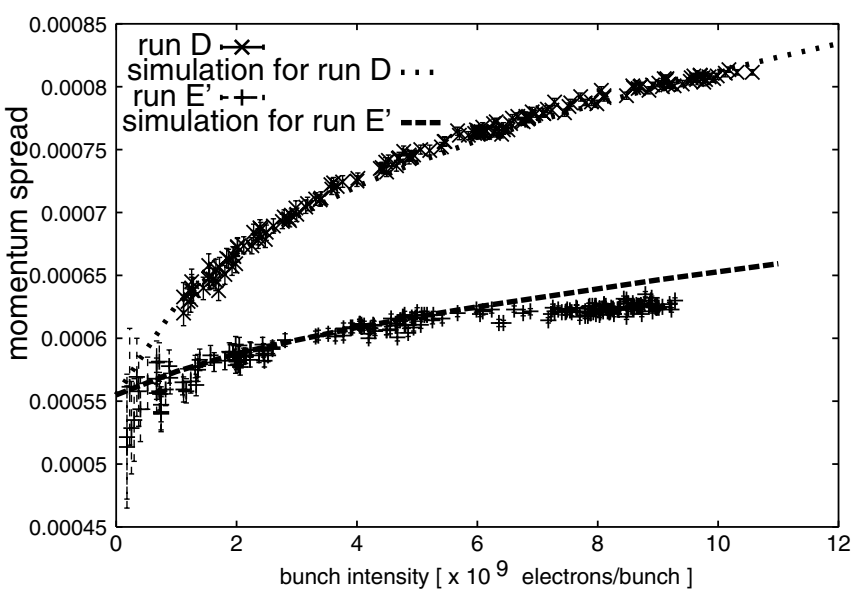

FIG. 4. Current dependence of the momentum spread: Data for the smallest (run D) and the largest emittance case (run $E^{\prime}$ ) are shown. ( $E^{\prime}$ means that the data were taken in same condition as $E$, but on another day.) The results of SAD simulations for $0.4 \%$ and $6 \%$ coupling are superimposed.

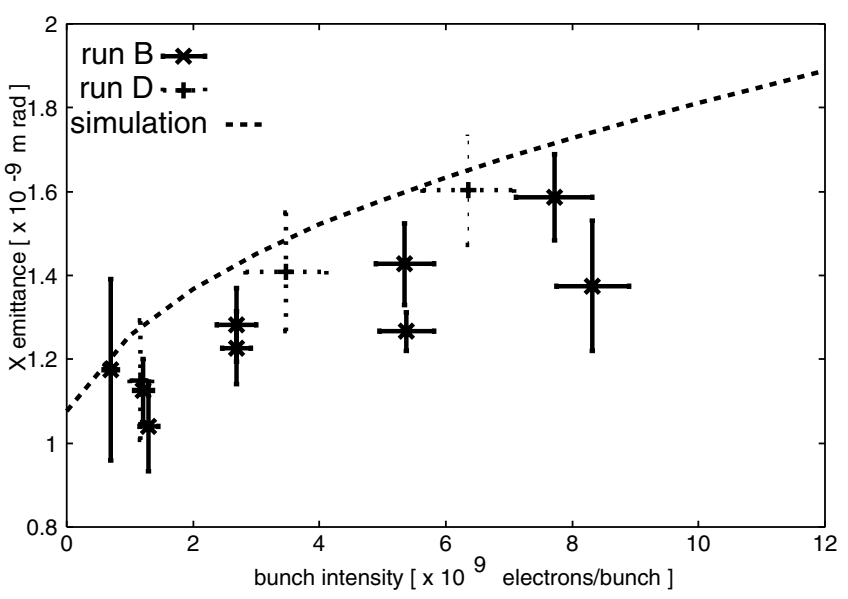

FIG. 3. Current dependence of the horizontal emittance: Data for the smallest emittance cases (runs B and D) are shown. The result of a SAD simulation for $0.4 \%$ coupling is superimposed.

was $4[\mathrm{pm} \mathrm{rad}]$ at beam energy of $1.3 \mathrm{GeV}$, which corresponds to the normalized emittance of $1.1 \times 1.0^{-8} \mathrm{~m}$. The vertical and horizontal emittances were 1.5 times bigger at $10^{10}$ [electrons/bunch] intensity than at zero current. No clear differences are seen between the strengths of the current dependence in the horizontal and the vertical plane. The simulation study shows that the expected ratio $\left[\left(\Delta \epsilon_{y}\right) /\left(\epsilon_{0 y}\right)\right] /\left[\left(\Delta \epsilon_{x}\right) /\left(\epsilon_{0 x}\right)\right]$ is about 1.6 if $\eta_{y}$ is dominant, and about 1 if the coupling is dominant [13]. The strength of the current dependence seems to be weaker in the cases with larger zero-current emittance ( $r_{10}$ of runs $E$ and $F$ in Table II). This is easily understood as the lower electron density reduces the rate of intrabeam scattering. It was found that our model calculation [14] reproduces well the overall characteristics of the data such as the current dependence of emittances, bunch length, and momentum spread.

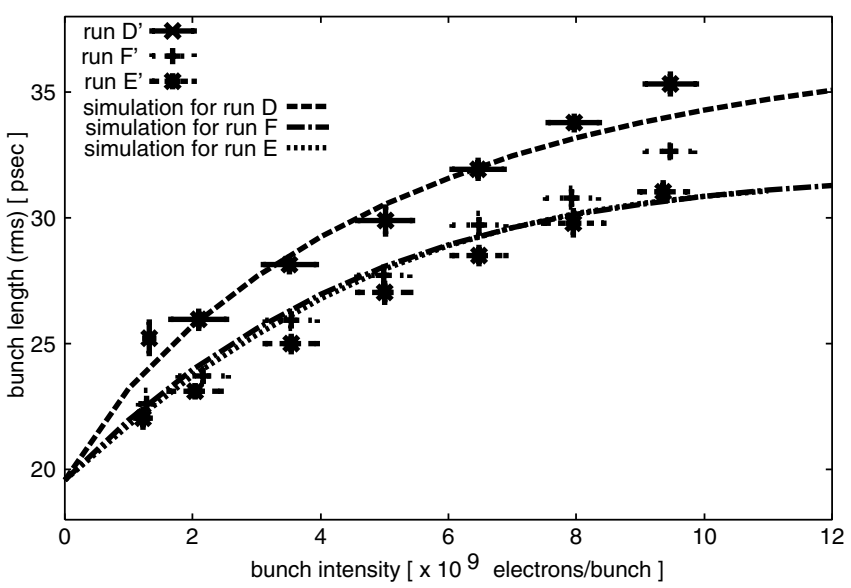

FIG. 5. Current dependence of the bunch length: Data are shown for the runs $D^{\prime}, F^{\prime}$, and $E^{\prime}$ (the symbols $D^{\prime}, E^{\prime}$, and $F^{\prime}$ indicate that the data were taken for the same condition as $D$, $E$, and $F$, but on another day.) The results of SAD simulations for $0.4 \%, 6 \%$, and $3 \%$ coupling are superimposed. 
We would like to note that the achieved vertical emittance in the single-bunch-mode operation satisfies the requirement of the present design of a linear collider. The emittance in multibunch-mode operation also has been measured [15] though a definite conclusion is yet to be reached.

The authors would like to thank Professors Y. Totsuka, K. Takata, Y. Kamiya, S. Iwata, and A. Enomoto of KEK for continuous support and encouragement. We also thank Professors E. Paterson and D. Burke of SLAC and K. Hubner of CERN for supporting the ATF project as an international collaboration.

[1] F. Hinode et al., KEK Internal Report No. 95-4, 1995.

[2] 2001 Report on the Next Linear Collider, 2001, http://www-project.slac.stanford.edu/lc/wkshp/ snowmass2001/; GLC Project Report, 2003, http:// lcdev.kek.jp/ProjReport/.

[3] H. Hayano (unpublished).

[4] M. Ross, J. Nelson, M. Woodley, and A. Wolski, SLACPUB-9239, 2002.
[5] H. Sakai et al., Jpn. J. Appl. Phys. 41, 6398 (2002).

[6] H. Sakai et al., Phys. Rev. ST Accel. Beams 5, 122801 (2002).

[7] Y. Honda et al. (unpublished).

[8] C. Steier et al., 2003 Particle Accelerator Conference, Oregon, 2003 (paper ID: RPPG018); H. Tanaka et al., in Proceedings of the Seventh European Particle Accelerator Conference, Vienna, 2000 (paper ID: MOP5A05).

[9] K. Kubo et al., Phys. Rev. Lett. 88, 194801 (2002).

[10] K. Kubo et al., in Proceedings of the Seventh European Particle Accelerator Conference, Vienna, 2000 (Ref. [8], pp. 483-484).

[11] K. Kubo, Phys. Rev. ST Accel. Beams 6, 092801 (2003).

[12] SAD is a computer program for accelerator design. http:// acc-physics.kek.jp/SAD/sad.html

[13] K. L.F. Bane et al., Phys. Rev. ST Accel. Beams 5, 084403 (2002).

[14] K. Kubo and K. Oide, Phys. Rev. ST Accel. Beams 4, 124401 (2001).

[15] Y. Honda et al., Phys. Rev. ST Accel. Beams 6, 092801 (2003). 